\title{
DESCARGAS EPILEPTIFORMES PERIÓDICAS LATERALIZADAS EM NEUROCISTICERCOSE
}

\author{
PAUZANES DE CARVALHO-FILHO:-ORLANDO MARTINS ARRUDA **; \\ SEBASTIATO EURICO DE MELO-SOUZA **
}

\begin{abstract}
RESUMO - Relatamos o caso de menina (10 anos) que apresentava sindrome psicorgânica subaguda associada a cefaléia, sem sinais patentes de hipertensão intracraniana. o diagnóstico de neurocisticercose foi firmado com base no exame do LCR. Foi tratada com praziquantel (50 $\mathrm{mg} / \mathrm{kg} /$ dia por 21 dias) e apresentou PLEDs sobre o hemisfério esquerdo no dia seguinte ao término do tratamento. Posteriormente, passou a apresentar sinais de disfunção córtico-subcortical desse mesmo lado, nos EEG subseqüentes. A TC inicialmente normal confirmou, com a evolução, maior comprometimento do hemisfério esquerdo, embora houvesse disseninasâo parenquimatosa bilateral de cisticercos. Kespondeu ben ao tratamento e cvoluiu com recuperação total dos sintomas. Discutimos, também, aspectos neurofisiológicos dessa anormalidade eletrencefalográfica.
\end{abstract}

\section{Periodic lateralized epileptiform discharges in neurocysticercosis}

SUMMARY — A case of a girl (10 years old) with a psycho-organic syndrome plus headache without signs of clear intracranial hypertension is related. The diagnosis of ncurocysticercosis was made by CSF abnormalities. PLEDs over the left cerebral hemisphere were recorded at the first day after treatment with praziquantel $(50 \mathrm{mg} / \mathrm{kg} / \mathrm{day}$ during 21 days). The first CT scan was normal and just after the treatment showed a prominent left cerebral hemisphere involvement, despite parenchymatous dissemination of cysticerci was bilateral. The treatment was successful and the patient became asymptomatic in the two years follow-up. The neurophysiologic aspects of this EEG abnormality is also discussed.

Chatrian e col. 3 chamaram de periodic lateralized epileptiform discharges (PLEDs)' quadro eletrencefalográfico composto por paroxismos de pontas, polipontas ou ondas agudas, que podem se seguir de onda lenta. Tem duração de fraçōes de segundo, com frequêencia de menos de uma a várias descargas por segundo, intercaladas por ritmo basal lentificado de baixa amplitude ou até mesmo não detectável e com ampla distribuição sobre um hemisfério cerebral. Esse quadro eletrencefalográfico tem sido observado em doenças cerebrais como acidentes vasculares cerebrais (AVC) $3,9,10$, traumatismo craniencefálico (TCE) $1,3,7$, encefalites 1,10 , abcesso $3,7,10$, tumores $1,3,8$, esclerose tuberosa 8,12 , anóxia 3,4 , epilepsias $3,6,7,8,12$, hematomas 9,10 , cisto perencefálico 12 e também em distúrbios metabólicos como diabetes 9 , alcoolismo ${ }^{9}$ e acidose metabólica 5. Virmani e col.11 descreveram o caso de um menino que apresentou PLEDs sobre o hemisfério direito no eletrencefalograma obtido dois meses antes de atingir o êxito letal. Clinicamente a criança apresentava-se em coma, com espasticidade nos 4 membros, hiperreflexia, reflexo cutâneo plantar em extensão bilateralmente, além de crises epilépticas predominantes no hemicorpo direito, de difícil controle clínico. O exame anatomopatológico mostrou numerosas vesiculas cisticercóticas em ambos os hemisférios cerebrais e cerebelo. A inexistência de explicaçăo adequăda para o aparecimento dessa anormalidade, assim como sua inespecificidade,

Trabalho realizado no Instituto de Neurologia de Goiania: * Neurologista (Endereço atual: Rua Goncalves Dias, $676 \multimap 78900$ Porto Velho RO); ** Neurologista. 
diminuem seu valor diagnóstico. No entanto, seu aparecimento sugere lesão estrutural importante tanto em nivel cortical quanto subcortical.

O caso que ora descrevemos, com diagnóstico confirmado pelo exame do líquido céfalo-raquidiano (LCR) e pela tomografia computadorizada do encéfalo (TC), teve um registro de PLEDs sobre o hemisfério esquerdo no dia seguinte ao término do tratamento com praziquantel. Apesar de ficar com lesão residual importante, principalmente sobre o hemisfério esquerdo, evoluiu bem num seguimento de dois anos.

\section{OBSERVACAO}

LOL, 10 anos, feminino, branca, natural de Guaraí-GO, admitida em 9-2-82 com aueixa de cefaléla holocraniana, episódios de vómitos e mudança de comportumento, tornando-se apática e relutante em responder às solicitações. Năo tinha antecedentes importantes nem relato de teníase. O exame neurológico era normal. A análise do LCR mostrava 1 célula/ $/ \mathrm{mm} 3,10 \mathrm{mg} \%$ de proteinas, $63 \mathrm{mg} \%$ de glicose; a reação de fixaçâo de complemento para cisticercose era negativa e a imunofluorescência para cisticercose era reator fracu. $\mathbf{A}$ TC foi considerada normal. O EEG de vigilia apresentava atividade predominante na faixa teta superposta por surtos de atividade delta difusa assincrona porém bilateral (Fig. 1). Houve

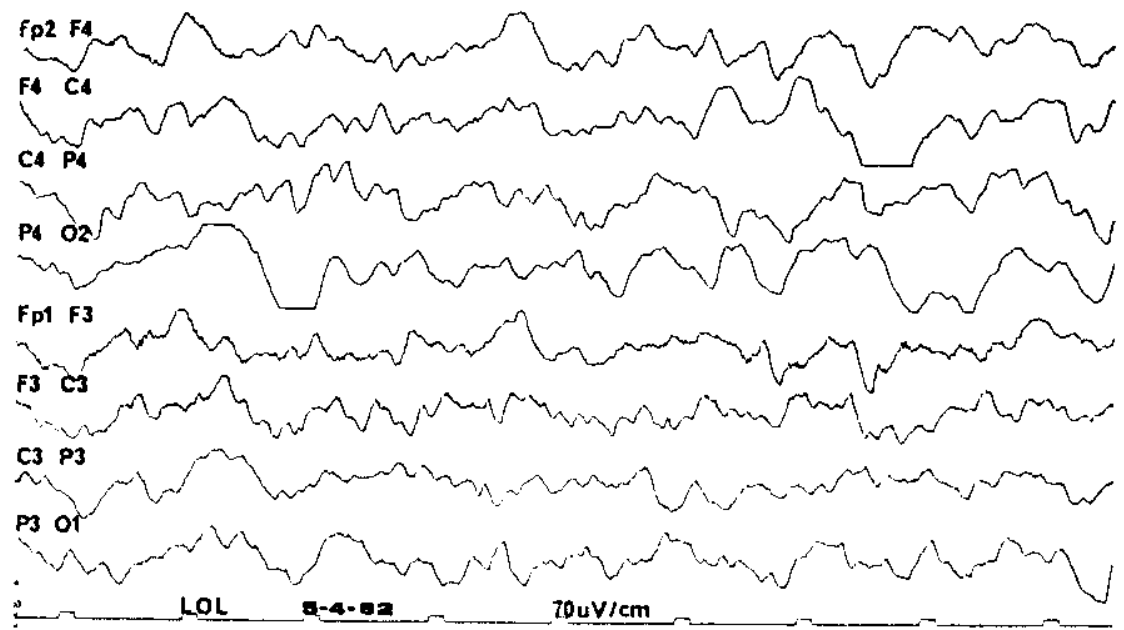

Fig. 1 - Cesso LOL. EEG: disfunção córtico-subcorticul difuș fsurtos de ondus lentas delta e tete irregulares).

melhora dos sintomas com prednisona e recebeu alta em uso de doses baixas desse medicamento com redução gradativa. Em 5-5-82 foi reinternada com intensa apatia; não respondia às solicitações verbais e apresentava episódios de agitação psicomotora. Esporadicamente queixava-se de cefaléia. O exame neurológico continuava sem anormalidades, exceto por ataxia de marcha e discutível estase venosa ao exame de fundo de olho. Nessa época, o exame radiológico de crânio continuava sem alteraçôes, o EEG com o mesmo padrão e uma nova TC ainda dentro dos padrōes de normalidade. O LCR no entanto apresentava: 13,6 célulus $/ \mathrm{mm}^{3}$ (5\% neutrófilos, $20 \%$ eosinófilos, $50 \%$ linfócitos e $25 \%$ reticulócitos), glicorracuia $61 \mathrm{mg} \%$ e proteinorraquia $25 \mathrm{mg} \%$; a eletroforese de proteinas do LCR apresentava $37 \%$ de gamaglobulinas; $\left.c^{\prime}\right\urcorner m$ o restante de seus componentes dentro dos padrōes de normalidade: as reaçōes de fixação de complemento e de imunofluorescencia para cisticercose eram positivas. Em 6-6-82 iniciamos tratamento com praziquantel na dose de $50 \mathrm{mg} / \mathrm{kg}$ por 21 dias. Houve acentuada piora clinica no segundo dia de tratamento. Entrou em coma com reaşāo extensora bilateral, apresentou crise epiléptica generalizada tônico-clónica sendo levada para o Centro de Tratamento Intensivo, no qual permaneceu por 6 dias, melhorando gradativamente. No dia seguinte ao término do tratamento, o EEG mostrou presença de PLEDs sobre o hemisferio esquerdo (Fig. 2). Durante todo o tratanento recebeu também dexametasona. Recebeu alta em 3-7-82, sem queixas importantes, sem usar anticonvulsivante e ainda usando doses baixas de dexametasona. Dois meses após a suspensão do corticóide ou seja, três 


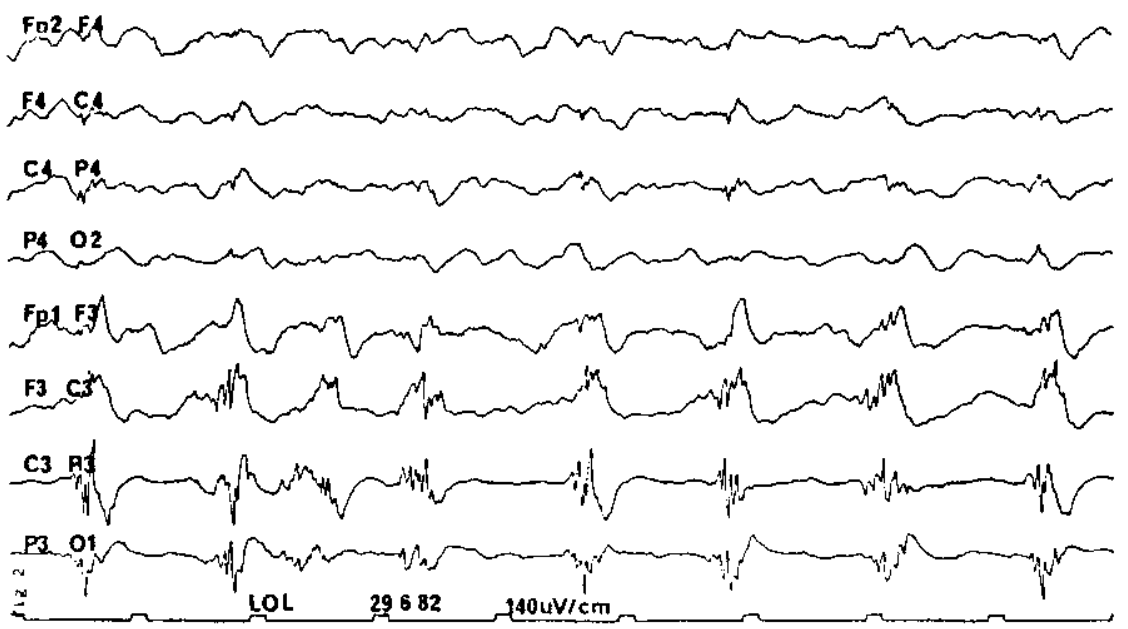

Fig. 2 - Caso LOL. EEG: PLEDs, paroxismos periódicos de múltiplas espiculas lateralizudiss, à esquerda.

meses após o término do tratamento, houve recrudescòncia da sintomatolngia. con cefaléia, distúrbio de comportamento, arressividade e crise epiléptica na véspera da consulta. Estava levemente cushingóide e apresentava assimetria de eflexos profundos, à esquerda mais vivos que à direita. Foj internada e submetida a novo ciclo de tratamento com praziquantel, sem a associaçāo de dexametasona. Năo houve piora clinica mas, pelo contrário, gradativa melhora dos sintomas. O exame do LCR não mostrou modificaçōes importantes nem antes nem depois do tratamento, permanecendo con citometria abaixo de 10 células/mm3, proteinas $\theta$ glicose normais e reações imunológicas para cisticercose positivas. O EES mostrava evidente prejuizo funcional sobre o hemisferio escuerdo (Fig. 3). A TC yassou a mnstrar pequenas

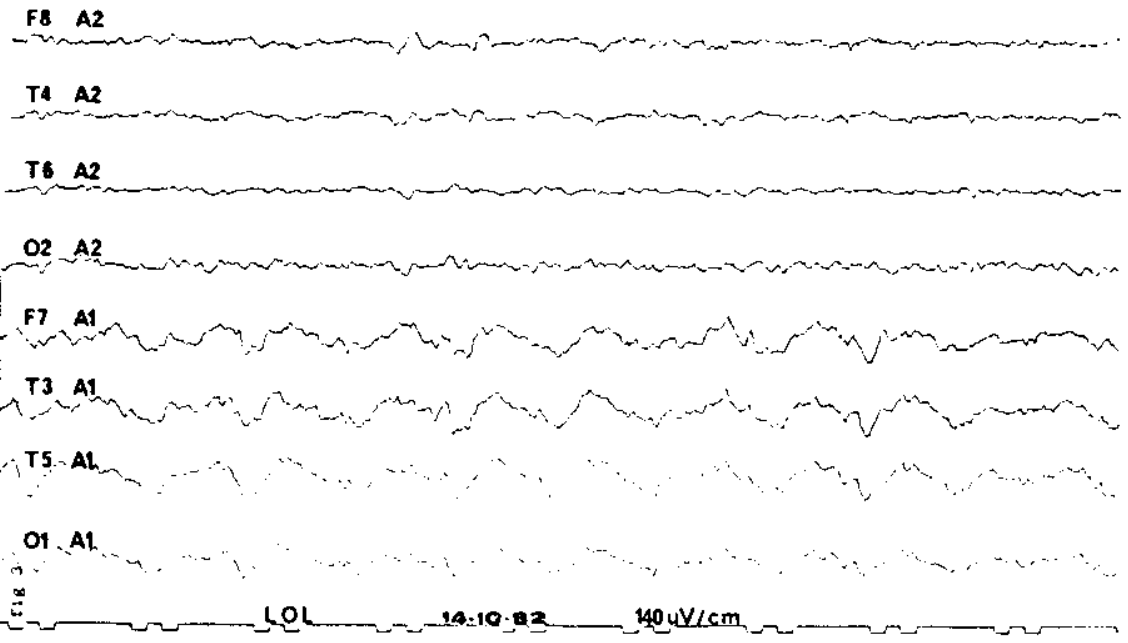

Fig. :- Caso LOL. EEG: atividade delta de repetição ritmica à esquerdr.

áreas de captação de contraste difusas sobre ambos os hemisférios cerebrais e discreta dilatacão vintriculur bilateral. Reavaliada em 11-3-83, relatava cefaléia esporádica de solucão espontânea, ainda com assimetria de reflexos profundos. $\mathrm{Em}$ 22-6-83 estava assintomática, freqüentando regularmente a escola. O EEG ainda mostrava evidente assimetria do traçado com redução da amplitude e lentificação sobre o hemisfério esquerdo (Fig. 4). A TC obtida 


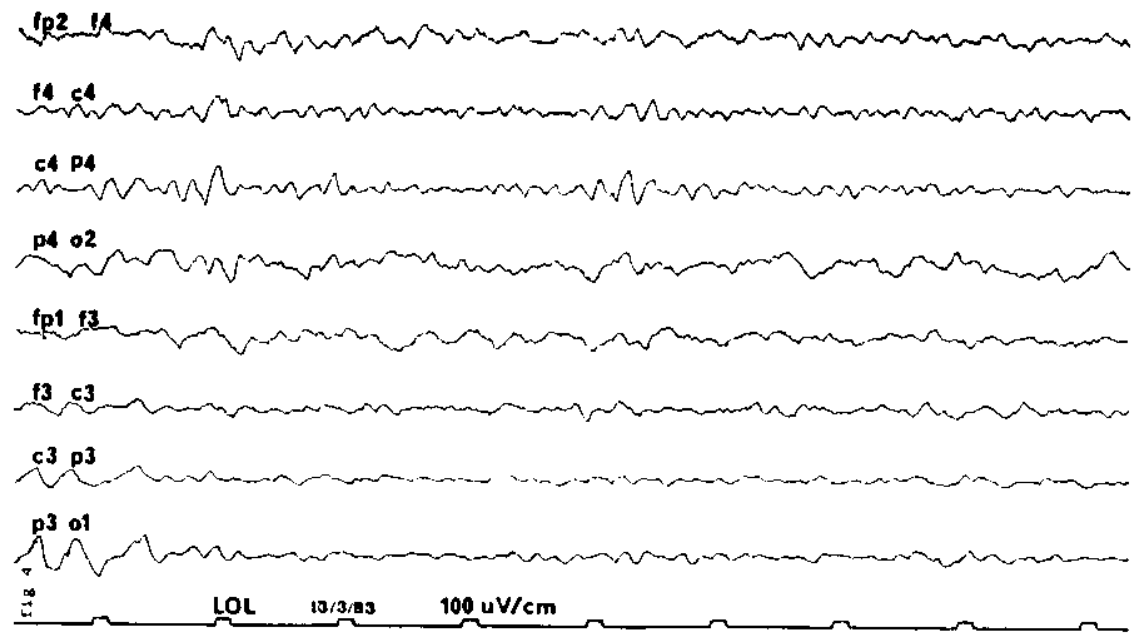

Fig. 4 - Caso LOL. EEG: atividade lenta, de 3 a $5 \mathrm{~Hz}$, predominando no hemisferio esquerdo $e$ nos quadrantes posteriores.

nessa época mostrava numerosas calcificações em ambos os hemisférios cerebrais, além dt atrofia cortical e subcortical predominando sobre as regióes temporal, opercular e parietal esquerda, com dilatação ventricular assimétrica (Fig. 5). Na última reavaliação, em 24-11-83, estava assintomática, com razoável desempenho escolar. O LCR mostrava 2,5 células/mm3, $10 \mathrm{mg} \%$ de proteínas, $72 \mathrm{mg} \%$ de glicose com provas imunológicas para cisticercose ainda positivas. O EEG e a TC năo mostravam mudanças significativas. Recebeu alta definitiva.

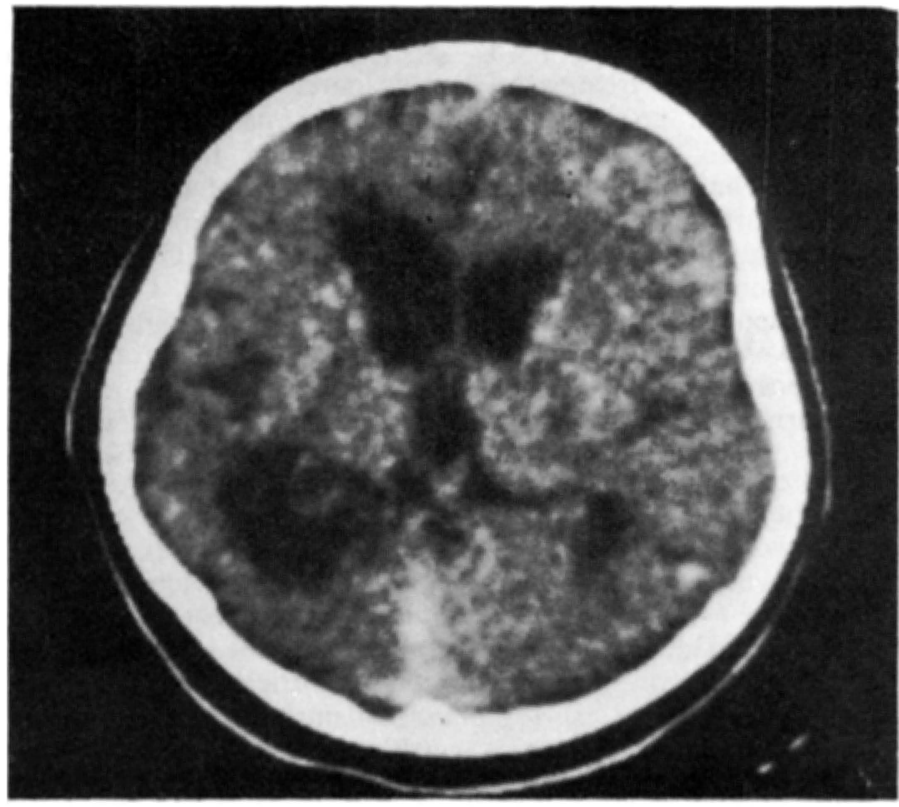

H'ig. 5-Caso LOL. Tomografia computadorizada de cranio com contraste: múltiplas pequenas calcificacões em ambos os hemisferios; perda de volume do hemisfertio esquerdo manifesta por dilatacão assimétrica dos ventrículos laterais (maior à esquerda) e da cisterna silviana; assimetria craniana; pequeno cisto no glomo do plexo coróide esquerdo. 
Os exames eletrencefalográficos foram realizados em aparçlho Nihen Khoden de $S$ canais, com eletrodos aplicados de acordo com sistema internaciona1 10-2C.

\section{COMENTARIOS}

O caso que ora descrevemos foi de diagnóstico difícil mesmo com o auxilio da TC, que só veio mostrar anormalidades significativas após o uso de praziquantel. $O$ diagnóstico foi firmado apenas com o LCR, depois que apareceram as alterações compativeis. A evolução mostrou que se tratava de uma disseminação maciça, em ambos os hemisférios, com acentuada piora clínica após o início do tratamento com o praziquantel. A evolução arrastada nos levou a considerar um novo ciclo de tratamento, que parece ter sido benéfico à paciente. Até onde sabemos foi a primeira paciente a receber praziquantel no estado de Goiás.

Após a crise epiléptica ocorrida no início do tratamento não foi realizado EEG, sendo o primeiro registro feito no final do tratamento, ocasião em que ocorreram PLEDs. E, portanto, possivel que seu aparecimento tenha sido mais precoce ou próximo ao aparecimento da crise epiléptica ou da instalação do estado de coma. Em nenhum de seus outros registros de EEG apareceram outros distúrbios epileptiformes. No caso semelhante registrado na literatura 11, oriundo da India, também uma criança, năo é feita referência ao exame do LCR e neurorradiológico. Clinicamente, as crises epilépticas eram dimidiadas à direita, apesar de no EEG registrar-se PLEDs também sobre o hemisfério direito. $O$ diagnóstico post-mortem mostrava encefalopatia difusa de etiologia neurocisticercótica.

PLEDs ocorrem em lesões parenquimatosas agudas, sub agudas ou crônicas que comprometem tanto o córtex quanto a substância branca, ou ambas. Na maioria dos casos, săo limitadas ao lado de maior envolvimento mas, podem ser bilaterais com predominio unilateral 3 . Em dois casos de Chatrian e col. 3 foram registrados PLEDs sobre os dois hemisférios mas independentes. Embora seja distúrbio tipicamente epileptiforme, é encontrado freqüentemente em doenças como AVC e abcesso cerebral, porém a manifestação clínica habitual são crises epilépticas $3,7,8,10,12$. Estas ocorreram enı $87,8 \%$ dos pacientes de Chatrian e col. 3 . As mais variadas formas de crises podem ser observadas, mas as mais freqüentes são as parciais simples e parcial continua 3 . Foram observadas também no pós-crise de epilepsia parcial complexa 6 . As crises associadas a esse achado são, de regra, resistentes ao tratamento habitual 3,8,11,12. Outras manifestações clínicas associadas, como déficit motor localizado, déficit sensitivo, assim como distúrbios da consciência, nos adultos, são frequentes 3 . Em crianças, podem ocorrer sem qualquer anormalidade importante 8 . Geralmente, não sofrem modificaçōes com o sono nem com os fatores de ativação 8. Costumam ser achados efêmeros, mas o grupo da Mayo Clinic relatou casos de registros de PLEDs que duraram até 20 anos 12 . Até o momento não há explicação para o aparecimento de PLEDs e nem demonstração satisfatória da sua origem. Enquanto existem inúmeros fatores sugerindo origem cortical, sāo observadas também em doenças exclusivas da substância branca ${ }^{3}$. A aplicação da lei da denervação parcial de Cannon, segundo a qual lesōes localizadas no córtex cerebral induzem a hiperexcitabilidade de áreas vizinhas, por interrupção de suas conexōes com outras áreas corticais, de acordo com o próprio Cannon, poderia justificar o aparecimento das descargas, mas não explica o desaparecimento das crises quando a lesão é removida cirurgicamente 3 . Isto tem lógica, desde que, a remoção da lesão nāo promoveria 'reinervaçāo' das áreas restantes. A 'autogenicidade cortical' é outra propriedade aventada para os padrōes periódicos associados à desconexão dos neurônios corticais em relação às áreas subcorticais 4. No entanto, apesar de estar esse distúrbio freqüentemente associado a lesōes orgânicas importantes, pode ele ser encontrado em distúrbios puramente metabólicos, como diabetes, alcoolismo e acidose metabólica 5.9. Depreende-se, portanto, que PLEDs sejam distúrbios funcionais de áreas acessiveis ao EEG, decorrentes de alguma propriedade residual de áreas lesadas ou de áreas vizinhas, às vezes sem uma necessária lesão estrutural, de caráter quase sempre transitório, porém, freqüentemente associadas a distúrbios funcionais persistentes. A inexistência de explicações satisfatórias nos induz a perseguir uma melhor compreensão do fenômeno. 


\section{REFERENCIAS}

1. Bertolucci PHF, Silva AB - Descargas epileptiformes periódicas lateralizadas: aspectos clínicos e eletrencefalográficos. Arq Neuro-Psiquiat (São Paulo) 45:364, 1987.

2. Cannon WB, Rosenblueth A - The Problem of «irritative foci» in central nervous system. Clin Eletroenceph 17:VIII, 1986.

3. Chatrian GE, Shaw CM, Leffman $\mathrm{H}$ - The significance of periodic lateralized epileptiform discharges in EEG: an electrographic, clinical and pathological study. Electroenceph Clin Neurophysiol $17: 177,1964$.

4. Janati A, Archer AL, Osteen PK - Coexistence of ectopic rhytms and periodic EEC pattern in anoxic encephalopathy. Clin Electroenceph 17:187, 1986.

5. Janati A, Chesser M, Husain MM - Periodic lacteralized epileptiform discharges (PLEDs): a possible role for metrbolic factors in pathogenesis. Clin Electroenceph 17:36, 1986.

6. McKeever M, Holmes GL, Russman BS - Feriodic lacteralized epileptiform discharges following a partial complex seizure. AM J EEG Technol 25:192, 1985.

7. Niedermeyer E, Lopes da Silva F - Electroencephalography. Basic Principles, Clinical Applications and Related Fields. Urban \& Schwarzenberg, Baltimore, 1982.

8. Pe Benito R, Cracco JB - Periodic lacteralized epileptiform discharges in infants and children. Ann Neurol 6:47, 1979.

9. Suter C, Creveling JG Jr - Periodic lacteralized epileptiform discharges. Electroenceph Clin Neurophysiol 20:624, 1966.

10. Torres F - Epilepsy: electroclinical correlation as a function of age. In Henry CE (ed): Current Clinical Neurophysiology: Update on EEG and Evoked Pontetials. Elsevier-North Holland, New York, 1980, pg 177.

11. Virmani V, Roy S, Kamala G - Periodic lacteralized epileptiform discharge in a case of diffuse cerebral cysticercosis. Neuropiidiatrie 8:196, 1977.

12. Westmoreland BF, Klass DW, Sharbrough FW - Chronic periodic lateralized epileptiform discharses, Arch Neurol 4s: $494,1986$. 